\title{
Levodopa-induced dyskinesia in Parkinson's disease: sleep matters
}

Running head: Role of sleep in levodopa-induced dyskinesia

Ninfa Amato ${ }^{1}$; Mauro Manconi ${ }^{1}$; Jens C. Möller ${ }^{2,3}$; Simone Sarasso ${ }^{4}$; Paolo Stanzione ${ }^{5}$; Claudio Staedler $^{1}$; Alain Kaelin-Lang ${ }^{1,6}$; Salvatore Galati ${ }^{1,6 *}$

${ }^{1}$ Movement Disorders Center, Neurocenter of Southern Switzerland, Lugano; ${ }^{2}$ Parkinson center, Zihlschlacht, Switzerland; ${ }^{3}$ Dept. of Neurology, Philipps-University Marburg, Germany;

${ }^{3}$ Department of Biomedical and Clinical Sciences "L. Sacco", Università degli Studi di Milano;

${ }^{4}$ Departimento di Medicina dei Sistemi, Università di Roma "Tor Vergata"; ${ }^{6}$ Università della Svizzera Italiana

*Correspondence to: Dr. Salvatore Galati, Neurocenter of Southern Switzerland,

Via Tesserete 46, 6903 Lugano. Tel +41 (0)91 8116921; Fax +41 (0)91 8116915

email: salvatore.galati@eoc.ch

Number of characters in the title: 59; in the running head: 39

Number of words in the Abstract 250; Introduction: 438, Discussion: 1495; and the body of the manuscript: 4489.

Number of figures: 7; color figures: 4; and tables: 4.

This article has been accepted for publication and undergone full peer review but has not been through the copyediting, typesetting, pagination and proofreading process, which may lead to differences between this version and the Version of Record. Please cite this article as doi: 10.1002/ana.25360 


\begin{abstract}
Objective. The spectrum of clinical symptoms changes during the course of Parkinson's disease. Levodopa therapy, while offering remarkable control of classical motor symptoms, causes abnormal involuntary movements as the disease progresses. These levodopa-induced dyskinesias (LIDs) have been associated with abnormal cortical plasticity. Since slow wave activity (SWA) of nonrapid eye movement (NREM) sleep underlies adjustment of cortical excitability, we sought to elucidate the relationship between this physiological process and LIDs.

Methods. Thirty-six patients at different stages of Parkinson's disease (PD) underwent wholenight video polysomnography-high-density EEG (vPSG-hdEEG), preceded by 1 week of actigraphy. To represent the broad spectrum of the disease, patients were divided into three groups by disease stage, (i) de novo (DNV; $\mathrm{n}=9$ ), (ii) advanced (ADV; $\mathrm{n}=13$ ), and (iii) dyskinetic (DYS; $\mathrm{n}=14$ ) and were compared to an age-matched control group (CTL; $\mathrm{n}=12$ ). The SWA-NREM content of the PSG-hdEEG was then temporally divided into 10 equal parts, from $\mathrm{T} 1$ to $\mathrm{T} 10$, and power and source analyses were performed. T2-T3-T4 were considered early sleep and were compared to T7-T8-T9, representing late sleep.

Results. We found that all groups, except the DYS group, manifested a clear-cut SWA decrease between early and late sleep.

Interpretation. Our data demonstrate a strong pathophysiological association between sleep and PD. Given that SWA may be a surrogate for synaptic strength, our data suggest that DYS patients do not have adequate synaptic downscaling. Further analysis is needed to determine the effect of drugs that can enhance cortical SWA in LIDs.
\end{abstract}




\section{Abbreviations}

$\mathrm{ADV}=$ advanced fluctuating patients; $\mathrm{AHI}=$ apnea-hypopnea index; AIMS = abnormal involuntary movement scale; CTL = healthy control subjects; DNV = de novo patients; DYS = advanced patients with dyskinesia; eSE = estimated sleep efficiency; eSL = estimated sleep latency; ESS = Epworth sleepiness scale; eTB = estimated time in bed; eTST = estimated total sleep time; $\mathrm{H} \& \mathrm{Y}=$ Hoehn and Yahr staging; iMAO = inhibitor of monoamine oxidase; LEDD = levodopa-equivalent daily dose; LID = levodopa-induced dyskinesia $;$ mBDI $=$ modified Beck depression scale; PD = Parkinson's disease; MDS-UPDRS = Movement Disorder Societysponsored revision of the Unified Parkinson Disease Rating Scale; MMSE = Mini-Mental State Examination; NREM = nonrapid eye movement; PSQI = Pittsburgh sleep quality index; RBD = REM sleep behavior disorder; REM = rapid eye movement; $r T M S=$ repetitive transcranial magnetic stimulation;; $\mathrm{SE}=$ sleep efficiency; SHY = synaptic homeostasis hypothesis; sLORETA = low-resolution brain electromagnetic tomography; SRBD = sleep-related breathing disorders; SWA = slow wave activity; TST = total sleep time; vPSG-hdEEG = video-highdensity EEG; WASO = wakefulness after sleep onset 


\section{Introduction}

Parkinson's disease (PD) is a neurodegenerative disorder presenting with a large spectrum of motor and nonmotor symptoms that are susceptible to change through the course of the disease, depending on the stage of disease progression and on pharmacological therapy ${ }^{1}$. Levodopa is a drug with strong and paradoxical effects on the course of PD; it successfully controls motor symptoms for several years and then induces motor fluctuation and abnormal involuntary movements, i.e., levodopa-induced dyskinesias (LIDs). This long-term drug-related complication causes important functional disability, often requiring complex pharmacological or surgical interventions.

Although LIDs are thought to be related to changes in neuronal plasticity in the striatal nuclei ${ }^{2}$, cortical changes are less well-known and are a new attractive area of research ${ }^{3}$. Studies involving repeated transcranial magnetic stimulation (rTMS) show abnormal motor cortex plasticity in patients with LID ${ }^{4,5}$. Furthermore, changes in cortical slow wave activity (SWA) have been observed in animal models of PD with LID ${ }^{6}$. This finding is of particular interest given that SWA during nonrapid eye movement (NREM) sleep is associated with fine adjustment of cortical excitability and plasticity, as postulated by the synaptic homeostasis hypothesis $(\mathrm{SHY})^{7-9}$. According to this hypothesis, daytime learning processes induce synaptic potentiation represented by an increase in synaptic strength. On the other hand, during the subsequent sleep, a consolidation of the learning process occurs together with a synaptic depotentiation represented by a downscaling of synaptic strength..$^{7-9}$ Moreover, SWA-NREM seems to act as a regulator of Hebbian plasticity, preventing saturation of the neuronal network by a sleep-related synaptic downscaling process ${ }^{10}$. Although the SHY requires further confirmation, the key role of sleep in brain plasticity is well supported ${ }^{11,12}$ by morphological 
evidence of sleep-dependent volumetric reduction of synaptic boutons ${ }^{7}$. Furthermore, druginduced potentiation of SWA with urethane reduced corticocortical and corticostriatal responses in vivo ${ }^{13}$. Additionally, rodents exposed to combined levodopa treatment and sleep deprivation developed earlier and more severe LID than animals that were not sleep deprived ${ }^{6}$. Because sleep disturbances are often part of the clinical spectrum of PD, even in the very early stages, we investigated the hypothesis that sleep and its effects on brain plasticity may influence the clinical phenotype of PD.

With the intent of translating the results previously obtained in animals ${ }^{6}$ to humans, we explored the correlation between objective sleep parameters and clinical features of different subpopulations of PD patients and age-matched controls. Using whole-night video polysomnography-high-density EEG (vPSG-hdEEG), we investigated possible locoregional differences in the homeostatic process that could be limited to discrete brain areas ${ }^{14}$. Some of the results presented here have already been published as a conference paper ${ }^{15}$.

\section{Materials and methods}

\section{Subjects}

All procedures were carried out with the appropriate understanding and written consent of the subjects and had previously been approved by the Local Ethics Committee (Cantonal Ethical Commission, reference number CE2562). This study was registered at ClinicalTrial.gov, reference number NCT02200887.

Thirty-six subjects with PD, according to the UK PD Society Brain Bank criteria ${ }^{16}$, were recruited for this study. Patients were divided into three groups, as previously described ${ }^{17}: 1$ ) de novo (DNV; $n=9)$, comprising patients with a recent diagnosis, naïve to dopaminergic therapy 
other than rasagiline; 2) advanced (ADV, $\mathrm{n}=13$ ), comprising patients not showing LID with their habitual therapy but demonstrating the end-of-dose or wearing-off phenomenon; and 3) dyskinetic (DYS, $\mathrm{n}=14$ ), comprising advanced patients experiencing motor fluctuations, showing LID, as confirmed by the enrolling physician.

In agreement with the diagnosis of PD, all DNV patients showed the typical clinical phenotype (e.g., asymmetrical parkinsonian syndrome, absence of atypical neurological findings) and normal cerebral magnetic resonance imaging, while an asymmetric dopaminergic deficit with [123I]-FPCIT (DATSCAN, GE Healthcare - Amersham Health) was observed in three of nine patients. Exclusion criteria were evidence for cognitive impairment, as defined by a Mini-Mental State Examination $(\mathrm{MMSE}) \leq 24$, and age $\geq 75$ years. Demographics and clinical characteristics of all patients are shown in Table 1. An age-matched control group, recruited from accompanying family members, nonclinical hospital staff, and volunteers, was also subjected to the same protocol $(\mathrm{CTL} ; \mathrm{n}=12)$.

Six of nine ADV and 10 of 11 DYS patients were administered a combination of levodopa and dopamine agonist treatment. The levodopa-equivalent daily dose (LEDD) was calculated according to the conversion formula reported elsewhere ${ }^{18}$. Two patients in each patient group (28.5\% DNV, 22.2\% ADV, and 18.1\% DYS) regularly took benzodiazepines. No CTL subjects took benzodiazepines.

One DNV patient was excluded for the subsequent development of progressive dementia, in the 6 months following the study, compatible with Lewy body dementia (Fig. 1 A). One DYS patient was excluded because she developed atypical clinical signs suggestive of multiple system atrophy with late-onset cardiovascular autonomic failure, urinary voiding disorder, and pyramidal signs, as previously described ${ }^{19}$. 


\section{Study design}

At the screening visit (Fig. 1A), mood and sleep complaints were investigated by means of the modified Beck depression scale (mBDI), Epworth sleepiness scale (ESS), and Pittsburgh sleep quality index (PSQI). All patients were assessed using the first three parts of the Movement Disorder Society-sponsored revision of the Unified Parkinson Disease Rating Scale (MDSUPDRS ${ }^{20}$ and Hoehn and Yahr (H\&Y) staging ${ }^{21}$. In addition, ADV and DYS patients were assessed using the fourth part of the MDS-UPDRS, while DYS patients were also evaluated with the Abnormal Involuntary Movement Scale (AIMS) ${ }^{22}$. After the screening visit, participants underwent a 1-week actigraphic recording (Fig. 1A). Then, participants arrived at the sleep center at 9:00 pm to prepare for the first habituation night in the sleep laboratory, which coincided with the last day of actigraphic recording. The following night, all patients underwent vPSG-hdEEG recording. The habituation night was included to reduce the "first-night effect" commonly associated with alterations in sleep architecture on the first night of sleep investigation, in comparison to subsequent nights ${ }^{23}$. All patients were followed in our outpatient service for a period of at least 6 months.

Patients were asked to adhere to regular sleep-wake schedules during the study. Antiparkinsonian medication was kept stable throughout the study. All ADV and DYS patients received levodopa treatment and other antiparkinsonian medications at a stable and optimized dose, as determined by the enrolling neurologists (S.G. and C.S.) for at least 4 weeks before the screening visit.

Based on sleep recordings, both patients and healthy volunteers were assessed for the presence of sleep-related breathing disorders (SRBDs). Since SRBDs are known to disrupt normal sleep 
architecture and to reduce NREM sleep duration ${ }^{24}$, five CTL individuals, three ADV patients, one DYS patient, and one DNV patient were excluded from further analysis because of an apnea-hypopnea index (AHI) > 5 (Fig. 1A).

For technical reasons, vPSG-hdEEG recording failed in one DNV and one ADV patient (Fig. 1A).

Therefore, seven of 12 CTL individuals, seven of nine DNV patients, nine of 13 ADV patients, and 11 of 14 DYS patients were included in the final sleep analysis. In addition, in two DNV patients and in one DYS patient, the vPSG-hdEEG recording was corrupted by artifacts (Fig. 1A) and excluded from the SWA analysis. Moreover, one DNV, one ADV, and one DYS patient did not undergo actigraphic monitoring due to technical failure.

\section{Data analysis}

\section{Actigraphy}

Wrist actigraphy has been established as a valid and reliable method for assessing the sleep-wake cycle ${ }^{25}$. A wristwatch-like device (Respironics Actiwatch 2, Philips, Best, The Netherlands) was attached to the subject's nondominant wrist and data were recorded continuously using 30-s sampling epochs. Actigraphy sleep data were scored by a validated algorithm included in the commercial software to obtain the estimated total sleep time (eTST), estimated time in bed (eTB), estimated sleep efficiency (eSE), and estimated sleep latency (eSL).

\section{Whole night video polysomnography-high-density EEG recording}

Nocturnal vPSG-hdEEG was performed in a standard sound-attenuated sleep laboratory room. Subjects were not allowed to drink caffeinated beverages 6 hours before the beginning of PSG 
and were allowed to sleep until their spontaneous awakening in the morning. Lights-out time was based on the individual's usual bed time and ranged between 10.30 and 11.30 p.m. All polysomnographic recordings included 256 EEG channels (Net Station System 200, v.4.0, Electrical Geodesics Inc., Eugene, OR, USA), submental electromyogram, electrooculogram, electrocardiogram, cardiorespiratory channels, and electromyogram of the right and left tibialis anterior muscles (bipolar derivations with two electrodes). The presence of REM sleep behavior disorder (RBD) was scored on the polysomnographic synchronized videotaped recording. Slow wave sleep (SWS) was defined as the deepest stage (N3) of NREM sleep. Sleep staging was performed according to standard scoring criteria, creating a monopolar montage with reference at the contralateral mastoid (A1 or A2), by an accredited clinical polysomnographist (M.M.), who was blind to the subject group to reduce the interscorer variability.

\section{$\underline{\text { EEG analysis }}$}

EEG data were sampled at $250 \mathrm{~Hz}$. Recordings were offline bandpass FIR filtered $(0.5-40 \mathrm{~Hz})$.

NREM sleep data were extracted, epochs containing arousals were excluded, and the remaining data were subdivided into 10 equal segments. We measured the percentage of $\mathrm{N} 3$ in each segment and selected the second segment (T2) as the first segment of early sleep to consider in order to observe a constant decline of SWS content because it contained the greater amount of N3 in each group of subjects or when pooled together. Sleep fragmentation during the falling asleep phase might be the reason for the delay in N3 peak expression. Therefore, the 2nd, 3rd, and $4^{\text {th }}$ segments and the $7^{\text {th }}, 8^{\text {th }}$, and $9^{\text {th }}$ segments were designated as early and late sleep, respectively, and analyzed further (Fig. 1B) using EEGLAB ${ }^{26}$ and custom-made MATLAB codes. The recordings were visually reviewed to exclude artifacts (total time excluded: $3.9 \%$ ). 
Bad channels were rejected and replaced using spherical interpolation. The fast Fourier transform (FFT) was separately calculated for the low $(1.5-2.0 \mathrm{~Hz})$ and high $(2.0-4.0 \mathrm{~Hz}) \delta$ frequency band, re-referenced to the average, downsampled (to $128 \mathrm{~Hz}$ ) 6-second EEG epochs with a 4-second overlap, and tapered with a Hamming window. Individual absolute band power was normalized to the total power. The SWA topographical distribution was obtained.

A source analysis was performed for each frequency band to obtain a 3D cortical distribution of the electric neuronal generators, using low-resolution brain electromagnetic tomography (sLORETA) ${ }^{27}$. The inverse solution was computed within a three-shell spherical head model, including the scalp, skull, and brain. The three-layer head model was coregistered to the Talairach human brain atlas ${ }^{28}$. The gray matter compartment was subdivided into 6340 voxels, with a spatial resolution of $5 \mathrm{~mm}$.

\section{$\underline{\text { Statistical analysis }}$}

Data were first examined for normal distribution using the Shapiro-Wilk test. Parametric data were assessed by one-way analysis of variance (ANOVA), and in case of significance, differences between pairs of groups were assessed by means of Tukey's post hoc tests. When not normally distributed, the Kruskal-Wallis test followed by a post hoc Mann-Whitney U test was used. Comparisons were considered statistically significant at a level of $P<0.05$. Correlation analyses were performed by means of the nonparametric Spearman's test. All tests were performed using IBM Statistics version 20 (IBM Inc., Armonk, NY, USA).

Differences in SWA in scalp topography among and between groups were investigated by means of a nonparametric method, based on permutation ${ }^{29}$, with false discovery rate (FDR) correction for multiple comparisons, where a common corrected threshold, identified as the last significant 
threshold, obtained using the Holms correction, was applied to all $P$ values. The same comparison was also performed on data from a reduced montage corresponding to the frontocentral areas.

Differences at the source level were investigated using sLORETA with a statistical nonparametric voxel-wise comparison within the CTL, DNV, ADV, and DYS groups for each frequency band. The level of significance was set at $P<0.05$.

All the results in the text, in the tables, and the figures are presented as the mean \pm SEM.

\section{Results}

\section{Demographics of control subjects and Parkinson's disease patients}

Demographic data are detailed in Table 1.

The mean H\&Y stage score was significantly lower for DNV than for ADV and DYS patients (Kruskal-Wallis, $\chi_{(2)}^{2}=16.787, P<0.001$; Mann-Whitney, DNV $<$ ADV: $U=4.00, \mathrm{Z}=-3.31$, $P<0.001 ; \mathrm{DNV}<\mathrm{DYS}: \mathrm{U}=4.50, \mathrm{Z}=-3.46, P<0.001)$.

The mean LEDD was significantly different among groups by one-way $\operatorname{ANOVA}\left(\mathrm{F}_{(2,27)}=\right.$ 25.365, $P=0.00$; Tukey's post hoc test: DNV > ADV: $P=0.003$; DNV $<$ DYS: $P<0.001$; ADV < DYS: $P=0.006)$.

The motor experiences of daily living (MDS-UPDRS II) showed a significant difference among the three patient groups (Kruskal-Wallis, $\chi_{(2)}^{2}=6.032, P=0.049$; at post hoc: $\mathrm{U}=12.00, \mathrm{Z}=$ 2.12, $P=0.34$; DNV < DYS). Regarding the motor assessment (MDS-UPDRS III) in the "on" state, no significant difference was observed among the patient groups.

\section{Subjective measures of depression and sleep quality}


All measurements are presented in Table 2. There was no difference among the four groups.

\section{Descriptive polysomnography data}

All descriptive sleep measures are presented in Table 3. There was no difference among the four groups.

RBD and/or REM without atonia were found in six of 27 patients (22.2\%): one DNV (14.2\%), three ADV (33.3\%), and two DYS (18.1\%) patients

\section{Correlation between sleep measures and clinical data}

Among the examined polysomnographic and clinical data, we found that TST, SE, and SWS showed significant correlations with disease duration and LEDD (Figs. 2 and 3).

In the patients as a whole $(\mathrm{n}=27)$, disease duration was negatively correlated with TST (Spearman's test, $\mathrm{r}_{\mathrm{s}}=-0.527, P=0.005$; Fig. 2A), but no such correlation was found when examining each group separately (DNV: $\mathrm{n}=7$; Spearman's test, $\mathrm{r}_{\mathrm{s}}=-0.250, P=0.589 ;$ ADV: $\mathrm{n}$ $=9 ; \mathrm{r}_{\mathrm{s}}=-0.517, P=0.154 ;$ DYS: $\mathrm{n}=11 ; \mathrm{r}_{\mathrm{s}}=-0.241, P=0.474 ;$ Fig. 2 A).

Similarly, in the patients as a whole $(\mathrm{n}=27)$, there was a negative correlation between disease duration and SE (Spearman's test, $\mathrm{r}_{\mathrm{s}}=-0.659, P<0.001$; Fig. 2B). When considering subgroups of patients, this correlation was maintained only in DYS patients $\left(n=11\right.$; Spearman's test, $r_{s}=-$ 0.664, $P=0.026)$, while no correlation between disease duration and SE was found in DNV $(\mathrm{n}=$ 7; Spearman's test, $\left.\mathrm{r}_{\mathrm{s}}=-0.143, P=0.760\right)$ or ADV patients $\left(\mathrm{n}=9\right.$; Spearman's test, $\mathrm{r}_{\mathrm{s}}=-0.333$, $P=0.381 ;$ Fig. 2B).

No significant correlation between disease duration and SWS was found in the whole patient group (n $=27$, Spearman's test, $\mathrm{r}_{\mathrm{s}}=0.043, P=0.823$; Fig. $2 \mathrm{C}$ ). However, when examining the 
three groups separately, we found that both DNV and ADV groups showed a clear positive correlation $\left(\mathrm{DNV} \mathrm{n}=7\right.$; Spearman's test, $\mathrm{r}_{\mathrm{s}}=0.786, P=0.036 ;$ ADV n $=9 ; \mathrm{r}_{\mathrm{s}}=0.783, P=$ 0.013; Fig. 2C), while the DYS group demonstrated a significant negative correlation between disease duration and SWS (n=11; Spearman's test, $\mathrm{r}_{\mathrm{s}}=-0.761, P=0.007$; Fig. 2C).

A negative correlation between TST and LEDD was observed in patients with motor fluctuations (ADV and DYS groups, $\mathrm{n}=20$, Spearman's test, $\mathrm{r}_{\mathrm{s}}=-0.475, P=0.026$; Fig. 3A). In these patients, LEDD was negatively correlated with SE (ADV and DYS, $n=20$; Spearman's test, $\mathrm{r}_{\mathrm{s}}=$ -0.495, $P=0.026$; Fig. 3B). When evaluating the patient groups individually, this negative correlation was still significant in the DYS group $\left(\mathrm{n}=11\right.$; Spearman's test, $\mathrm{r}_{\mathrm{s}}=-0.633, P=$ 0.036; Fig. 3B) but not in the ADV group $\left(\mathrm{n}=9\right.$; Spearman's test, $\left.\mathrm{r}_{\mathrm{s}}=0.000, P=1.000\right)$.

Moreover, LEDD was negatively correlated with SWS only in the DYS group $(\mathrm{n}=11$; Spearman's test, $\left.\mathrm{r}_{\mathrm{s}}=-0.682, P=0.021\right)$, while no SWS-LEDD correlation was observed in the $\operatorname{ADV}\left(\mathrm{n}=9\right.$; Spearman's test, $\left.\mathrm{r}_{\mathrm{s}}=0.077, P=0.845\right)$ and combined ADV and DYS groups $(\mathrm{n}=$ 20; Spearman's test, $\left.\mathrm{r}_{\mathrm{s}}=-0.302, P=0.195\right)$ (Fig. 3C).

\section{Descriptive actigraphy data}

All the descriptive actigraphic measures are presented in Table 3. All the measures were similar in all four groups (CTL, DNV, ADV, DYS).

\section{Dyskinesia and motor fluctuations correlate inversely with eTST}

We found a negative correlation between eTST and AIMS (DYS: Spearman's test, $n=9 ; r_{s}=-$ 0.733, $P=0.035)$ and between eTST and MDS-UPDRS IV (DYS: Spearman's test, $\mathrm{n}=9 ; \mathrm{r}_{\mathrm{s}}=-$ 0.817, $P=0.007 ;$ Fig. 4A). 
We did not observe any correlation of the AIMS and MDS-UPDRS IV scores with eSE (DYS: Spearman's test, $\mathrm{n}=9 ; \mathrm{r}_{\mathrm{s}}=-0.536, P=0.137$ and $\mathrm{r}_{\mathrm{s}}=-0.221, P=0.567$, respectively; Fig. 4B).

\section{Changes in slow-wave activity during early and late sleep}

In early sleep, control subjects showed a significantly greater amount of SWA, diffused over the whole scalp, compared to PD patients $(P<0.01$; FDR adjusted $)$, as a whole group and separately. Moreover, delta power was greater in DNV compared to the other patients' groups $(P$ $<0.01$; FDR adjusted) and in ADV compared to DYS $(P<0.01$; FDR adjusted), with the DYS group having the lowest content of SWA. As expected, the decrease was mainly in the high $\delta$ frequency band $(2.0-4.0 \mathrm{~Hz})^{30}$ (Fig. 5A-C). When comparing within groups, we found a significant difference between early and late sleep in the CTL $(n=7)$, DNV $(n=5)$, and ADV groups (n = 9; $P<0.01$; FDR adjusted) but not in the DYS group $(\mathrm{n}=10$, Fig. 5B).

In late sleep, when contrasting between groups selecting only frontocentral channels, delta power was lower in DNV compared to the other groups $(P<0.01 ;$ FDR adjusted $)$ and in ADV compared to DYS $(P<0.01$; FDR adjusted), with the DYS group having the greatest content of SWA among PD patients.

The voxel-wise comparison (sLORETA) between groups showed a decrease in SWA between early and late sleep, reaching significance only in the CTL group (n $=7 ; P<0.01)$, localized over frontocentral regions (Brodmann areas: 4, 6, 13, 24, 31, Fig. 6).

\section{Discussion}

Levodopa is currently the most effective available treatment for motor symptoms in PD, but its use is complicated by the development of motor fluctuations and LID. These abnormal 
movements are mild at the beginning but become disabling as the disease progresses, interfering with quality of life and being a source of morbidity. The pathogenesis of LID is largely unknown, but the onset of PD symptoms at an earlier age ${ }^{31}$, disease duration ${ }^{32}$, and higher cumulative dosage of levodopa ${ }^{33}$ are well-known risk factors for LID development. The involvement of age at onset suggests an underlying genetic background for a dysfunction in brain plasticity, as confirmed by several experiments ${ }^{34}$.

Sleep has a function in modulating brain plasticity ${ }^{11,12}$, but its characteristics in relation to the clinical phenotype of PD have not yet been addressed.

Herein, we provided evidence of a close relationship between sleep and several clinical features in a population of patients with PD at different stages, afflicted by motor fluctuations with or without LID. We show the correlation of sleep architecture with disease duration and LEDD and of actigraphic data with the severity of dyskinesia. Importantly, we found an overnight physiological decline in SWA in CTL, DNV, and ADV subjects but not in DYS subjects, who showed a persistent level of SWA throughout the night.

Polysomnographic studies of PD have shown conflicting results ${ }^{35}$. For instance, some authors have described changes in SE, TST, SL, and sleep stages in PD patients compared to agematched healthy controls, ${ }^{36}$, while others have not found significant differences ${ }^{37}$. However, compared to these previous studies, in this study, we examined sleep architecture with respect to the disease stage and with respect to the presence/absence of motor fluctuations and LID. We would suggest that this patient classification allows a more accurate detection of those differences that would otherwise not be clearly defined in a heterogeneous group. For instance, in the whole PD patient cohort, we found that both the TST and SE were negatively correlated with disease duration, as has been already described and that while the correlation between SWS 
and disease duration was positive in both DNV and ADV patients, it was surprisingly negative in DYS patients. Diederich and colleagues (2005) found a negative correlation between SWS and disease duration, but they did not take the presence of motor fluctuations or LID into account. Since the patient sample was older and featured a longer disease duration than our population, it is likely that they investigated mainly advanced PD patients ${ }^{38}$. Although far from conclusive, the positive correlation between SWS and disease duration in both DNV and ADV patients might reflect compensatory mechanisms within the SHY framework aimed at maintaining an appropriate homeostatic process, which could be ineffective in DYS patients. However, the amount of SWS does not directly reflect a more efficient SWA-mediated downscaling process ${ }^{14}$. As mentioned above, the risk of developing dyskinesia or motor fluctuations is closely linked to the levodopa cumulative dose ${ }^{33}$. Therefore, we extended our correlation analysis to sleep parameters and LEDD. We found an intriguing negative correlation of TST and SE with LEDD in all patients with motor fluctuations (ADV and DYS groups). Notably, SWS was negatively correlated with LEDD only in patients demonstrating LID. The impact of levodopa therapy on sleep is not well defined and has been the subject of only few studies. Levodopa, in fact, may have a direct effect on sleep macrostructure or may improve sleep by improving motor nocturnal performance. In a small sample of PD patients, reduced SWS and REM sleep with prolonged SL and WASO was observed following the initiation of levodopa treatment. ${ }^{39}$ However, some studies ${ }^{40}$ conducted in larger populations of patients did not detect significant changes in SWS and REM sleep, although both SL and WASO were reduced after levodopa treatment. Similar to our findings, a case-control polysomnographic study revealed reduced TST associated with increased LEDD ${ }^{36}$, although motor fluctuations and LID were not assessed. 
Although association does not imply causality, the notion of cumulative LEDD as a risk factor for LID suggests that levodopa use, especially in a day-night pulsatile manner, could be the cause of reduced TST, subsequently enhancing LID. In fact, our 1-week actigraphy data demonstrated a clear negative correlation between eTST and the severity of dyskinesia. Indeed, poor nighttime sleep has been positively associated with LID in a recent study with a large cohort of patients ${ }^{41}$. Along these lines, sleep deprivation has been shown to enhance LID in a rodent model ${ }^{6}$ and in PD patients ${ }^{42}$. On the other hand, subjective clinical amelioration after sleep is frequently mentioned by patients, mainly in those with long disease duration and motor fluctuations ${ }^{43,44}$.

The third main finding of our study concerns SWA. Notably, all groups, except the DYS group, manifested a clear-cut physiological decrease in SWA between early and late sleep. The degree of overnight SWA reduction was notable in CTL individuals, in whom we also found early-late sleep differences in the source reconstruction analysis. The early-late difference was still significant in both the DNV and ADV groups.

The lack of a significant difference between early-late SWA in DYS patients could be due either to impaired downscaling or to a lower buildup in these patients. Indeed, DYS patients, start from a lower early SWA level compared to the rest of ADV and DNV subjects. However, the observed SWA reduction in early sleep could be a consequence of a chronic deficit of synaptic downscaling during the night in dyskinetic patients. ${ }^{41}$ In these respects, chronic sleep deprivation has been associated with an impairment of synaptic potentiation. ${ }^{45}$

We are inclined to believe that our results support a disruption of homeostatic process per se. In fact, DNV patients have late sleep SWA content lower than ADV and DYS patients, and, even more important to our argument against a flooring effect, lower than CNT participants. 
Moreover, DYS patients showed a greater amount of SWA in the late sleep compared to the other groups. Therefore, if our results were due to an overall SWA impairment and to a floor effect rather than to a disrupted homeostatic process, we would not have expected to observe the lowest level of early sleep SWA in DYS together with the highest level of late sleep SWA in these patients, but rather a similar content of SWA in the late sleep among the groups.

Since dyskinesia is a motor phenomenon, the same analysis performed selecting only the frontal channels heightened the difference between DYS and ADV, and the alteration of homeostatic processes is also confirmed by the results obtained using a frontal low-density EEG channel montage.

The SWA analysis allowed the recognition of differences between CTL individuals and PD patients as well as between advanced patients with or without LID. None of the participants had cognitive impairment, which is usually related to the presence of cortical pathology ${ }^{46}$. Therefore, our results suggest that cortical SWA changes may be associated with the development of LID rather than with a structural pathology.

Levodopa-treated dyskinetic rats manifest aberrant corticostriatal synaptic plasticity that impaired the ability of the striatum to discriminate between relevant and irrelevant cortical inputs ${ }^{2}$. From the SHY prospective, the homeostatic changes of net synaptic strength across the sleepwake cycle define the threshold for associative plasticity; therefore, a reduction of sleep-related synaptic downscaling can hide an abnormal saturation of the corticostriatal network.

Certainly, our findings related to cortical plasticity do not exclude an impaired top-down corticostriatal input, which induced pathological plasticity in the striatum ${ }^{47}$, or even that an intrinsic trait in cortical plasticity may predispose a subgroup of patients to LID. 
Supporting this view, normal motor-skill learning and synaptic plasticity require normal dopaminergic input within the primary motor cortex (M1) ${ }^{13}$. Dopamine projections to the cortex arise mainly from the ventral tegmental area (VTA) ${ }^{48}$, which is involved later in the course of the disease ${ }^{49}$. The timing of the neurodegenerative process that is reflected by the dopamine content in the cortex might underlie the impaired plasticity and the subsequent development of LID $^{4,5}$. Of note, several lines of evidence have linked LID with impulse-control disorders, which are notoriously associated with VTA dysfunction ${ }^{50}$.

In conclusion, these results support our preclinical findings of a clear association between sleep and LID at the electrophysiological, behavioral, and biochemical levels.

Although our findings do not imply a causative role for the lack of SWA reduction in the emergence of LID, in light of the SHY framework, they do suggest an association between sleep and some clinical phenotypes of PD and suggest a relationship between sleep disruption and LID. Additional studies are warranted to establish a causative relationship between an abnormal sleep-related downscaling process and LID development. The small size of our sample, although homogenous and well characterized, represented a limitation of our study, and a larger confirmatory study is needed to support this theory, which will pave the way for pioneering SWA-enhancing therapies in PD.

\section{Acknowledgements}

We would like to express our most sincere gratitude to the participants who devoted their time and efforts to take part in this study. We would like to thank Chiara Prosperetti and Serena 
Caverzasio for their critical reading. We are particularly grateful to Lorenzo Toffolet for his technical assistance.

We thank the Scientific Research Advisory Board of Ente Ospedaliero Cantonale (ABREOC) and 'Fondazione per lo studio delle malattie neurodegenerative delle persone adulte e dell'anziano del Ticino' for financial support to C.S and S.G.

\section{Author Contributions}

SG, PS, and JCM contributed to the conception and design of the study; NA, MM, and SS contributed to acquisition and analysis of the data; and AKL, SG, and NA contributed to drafting the manuscript and figures.

\section{Potential Conflicts of Interest}

Nothing to report.

\section{References}

1. Galati S, Stefani A. Deep brain stimulation of the subthalamic nucleus: All that glitters isn't gold? Mov. Disord. Off. J. Mov. Disord. Soc. 2015;30(5):632-637.

2. Calabresi P, Filippo MD, Ghiglieri V, et al. Levodopa-induced dyskinesias in patients with Parkinson's disease: filling the bench-to-bedside gap. Lancet Neurol. 2010;9(11):11061117.

3. Rajan R, Popa T, Quartarone A, et al. Cortical plasticity and levodopa-induced dyskinesias in Parkinson's disease: Connecting the dots in a multicomponent network. Clin. Neurophysiol. 2017;128(6):992-999.

4. Huang Y-Z, Rothwell JC, Lu C-S, et al. Abnormal bidirectional plasticity-like effects in Parkinson's disease. Brain J. Neurol. 2011;134(Pt 8):2312-2320.

5. Morgante F, Espay AJ, Gunraj C, et al. Motor cortex plasticity in Parkinson's disease and levodopa-induced dyskinesias. Brain J. Neurol. 2006;129(Pt 4):1059-1069. 
6. Galati S, Salvade A, Pace M, et al. Evidence of an association between sleep and levodopainduced dyskinesia in an animal model of Parkinson's disease. Neurobiol. Aging 2015;36(3):1577-1589.

7. de Vivo L, Bellesi M, Marshall W, et al. Ultrastructural evidence for synaptic scaling across the wake/sleep cycle. Science 2017;355(6324):507-510.

8. Riedner BA, Vyazovskiy VV, Huber R, et al. Sleep homeostasis and cortical synchronization: III. A high-density EEG study of sleep slow waves in humans. Sleep 2007;30(12):1643-1657.

9. Tononi G, Cirelli C. Sleep and the Price of Plasticity: From Synaptic and Cellular Homeostasis to Memory Consolidation and Integration. Neuron 2014;81(1):12-34.

10. Turrigiano GG, Nelson SB. Homeostatic plasticity in the developing nervous system. Nat. Rev. Neurosci. 2004;5(2):97-107.

11. Poe GR. Sleep Is for Forgetting. J. Neurosci. 2017;37(3):464-473.

12. Sara SJ. Sleep to Remember. J. Neurosci. 2017;37(3):457-463.

13. Galati S, Wei S, Orban G, et al. Cortical slow wave activity correlates with striatal synaptic strength in normal but not in Parkinsonian rats. Exp. Neurol. 2017;

14. Huber R, Ghilardi MF, Massimini M, Tononi G. Local sleep and learning. Nature 2004;430(6995):78-81.

15. Galati S, Sarasso S, Moeller C, et al. Synaptic homeostasis in Parkinson's disease: An highdensity Eeg study in different stage of the disease. Mov. Disord. 2016;31:S276.

16. Gibb WR, Lees AJ. The significance of the Lewy body in the diagnosis of idiopathic Parkinson's disease. Neuropathol. Appl. Neurobiol. 1989;15(1):27-44.

17. Lunardi G, Galati S, Tropepi D, et al. Correlation between changes in CSF dopamine turnover and development of dyskinesia in Parkinson's disease. Parkinsonism Relat. Disord. 2009;15(5):383-389.

18. Tomlinson CL, Stowe R, Patel S, et al. Systematic review of levodopa dose equivalency reporting in Parkinson's disease. Mov. Disord. Off. J. Mov. Disord. Soc. 2010;25(15):2649-2653.

19. Calandra-Buonaura G, Guaraldi P, Sambati L, et al. Multiple system atrophy with prolonged survival: is late onset of dysautonomia the clue? Neurol. Sci. 2013;34(10):18751878.

20. Goetz CG, Fahn S, Martinez-Martin P, et al. Movement Disorder Society-sponsored revision of the Unified Parkinson's Disease Rating Scale (MDS-UPDRS): Process, format, and clinimetric testing plan. Mov. Disord. Off. J. Mov. Disord. Soc. 2007;22(1):41-47. 
21. Hoehn MM, Yahr MD. Parkinsonism: onset, progression, and mortality. 1967. Neurology 1998;50(2):318 and 16 pages following.

22. Goetz CG, Damier P, Hicking C, et al. Sarizotan as a treatment for dyskinesias in Parkinson's disease: a double-blind placebo-controlled trial. Mov. Disord. Off. J. Mov. Disord. Soc. 2007;22(2):179-186.

23. Agnew HW, Webb WB, Williams RL. The first night effect: an EEG study of sleep. Psychophysiology 1966;2(3):263-266.

24. Fietze I, Quispe-Bravo S, Hänsch T, et al. Arousals and sleep stages in patients with obstructive sleep apnoea syndrome: Changes under nCPAP treatment. J. Sleep Res. 1997;6(2):128-133.

25. Ancoli-Israel S, Cole R, Alessi C, et al. The role of actigraphy in the study of sleep and circadian rhythms. Sleep 2003;26(3):342-392.

26. Delorme A, Makeig S. EEGLAB: an open source toolbox for analysis of single-trial EEG dynamics including independent component analysis. J. Neurosci. Methods 2004;134(1):921.

27. Pascual-Marqui RD, Esslen M, Kochi K, Lehmann D. Functional imaging with lowresolution brain electromagnetic tomography (LORETA): a review. Methods Find. Exp. Clin. Pharmacol. 2002;24 Suppl C:91-95.

28. Talairach J, Tournoux P. Co-planar stereotaxic atlas of the human brain: 3-dimensional proportional system: an approach to cerebral imaging. Stuttgart; New York: Georg Thieme; 1988.

29. Nichols TE, Holmes AP. Nonparametric permutation tests for functional neuroimaging: a primer with examples. Hum. Brain Mapp. 2002;15(1):1-25.

30. Achermann P, Borbély AA. Low-frequency $(<1 \mathrm{~Hz})$ oscillations in the human sleep electroencephalogram. Neuroscience 1997;81(1):213-222.

31. Ku S, Glass GA. Age of Parkinson's disease onset as a predictor for the development of dyskinesia. Mov. Disord. Off. J. Mov. Disord. Soc. 2010;25(9):1177-1182.

32. Schrag A, Quinn N. Dyskinesias and motor fluctuations in Parkinson's disease. A community-based study. Brain J. Neurol. 2000;123 ( Pt 11):2297-2305.

33. Warren Olanow C, Kieburtz K, Rascol O, et al. Factors predictive of the development of Levodopa-induced dyskinesia and wearing-off in Parkinson's disease. Mov. Disord. Off. J. Mov. Disord. Soc. 2013;28(8):1064-1071.

34. Linazasoro G. New ideas on the origin of L-dopa-induced dyskinesias: age, genes and neural plasticity. Trends Pharmacol. Sci. 2005;26(8):391-397. 
35. Peeraully T, Yong M-H, Chokroverty S, Tan E-K. Sleep and Parkinson's disease: a review of case-control polysomnography studies. Mov. Disord. Off. J. Mov. Disord. Soc. 2012;27(14):1729-1737.

36. Yong M-H, Fook-Chong S, Pavanni R, et al. Case control polysomnographic studies of sleep disorders in Parkinson's disease. PloS One 2011;6(7):e22511.

37. Diederich NJ, Rufra O, Pieri V, et al. Lack of polysomnographic Non-REM sleep changes in early Parkinson's disease. Mov. Disord. Off. J. Mov. Disord. Soc. 2013;28(10):14431446.

38. Diederich NJ, Vaillant M, Mancuso G, et al. Progressive sleep "destructuring" in Parkinson's disease. A polysomnographic study in 46 patients. Sleep Med. 2005;6(4):313318.

39. Kales A, Ansel RD, Markham CH, et al. Sleep in patients with Parkinson's disease and normal subiects prior to and following levodopa administration. Clin. Pharmacol. Ther. 1971;12(2part2):397-406.

40. Ferreira T, Prabhakar S, Kharbanda P. Sleep disturbances in drug naïve Parkinson's disease (PD) patients and effect of levodopa on sleep. Ann. Indian Acad. Neurol. 2014;17(4):416.

41. Mao C-J, Yang Y-P, Chen J-P, et al. Poor nighttime sleep is positively associated with dyskinesia in Parkinson's disease patients. Parkinsonism Relat. Disord. 2018;48:68-73.

42. Bertolucci PH, Andrade LA, Lima JG, Carlini EA. Total sleep deprivation and Parkinson disease. Arq. Neuropsiquiatr. 1987;45(3):224-230.

43. Currie LJ, Bennett JP, Harrison MB, et al. Clinical correlates of sleep benefit in Parkinson's disease. Neurology 1997;48(4):1115-1117.

44. Sherif E, Valko PO, Overeem S, Baumann CR. Sleep benefit in Parkinson's disease is associated with short sleep times. Parkinsonism Relat. Disord. 2014;20(1):116-118.

45. Campbell IG, Guinan MJ, Horowitz JM. Sleep deprivation impairs long-term potentiation in rat hippocampal slices. J. Neurophysiol. 2002;88(2):1073-1076.

46. Kingsbury AE, Bandopadhyay R, Silveira-Moriyama L, et al. Brain stem pathology in Parkinson's disease: an evaluation of the Braak staging model. Mov. Disord. Off. J. Mov. Disord. Soc. 2010;25(15):2508-2515.

47. Calabresi P, Pisani A, Rothwell J, et al. Hyperkinetic disorders and loss of synaptic downscaling. Nat. Neurosci. 2016;19(7):868-875.

48. Hosp JA, Pekanovic A, Rioult-Pedotti MS, Luft AR. Dopaminergic projections from midbrain to primary motor cortex mediate motor skill learning. J. Neurosci. Off. J. Soc. Neurosci. 2011;31(7):2481-2487. 
49. German DC, Manaye K, Smith WK, et al. Midbrain dopaminergic cell loss in Parkinson's disease: computer visualization. Ann. Neurol. 1989;26(4):507-514.

50. Voon V, Napier TC, Frank MJ, et al. Impulse control disorders and levodopa-induced dyskinesias in Parkinson's disease: an update. Lancet Neurol. 2017;16(3):238-250.

Table 1 Demographics of cohorts

\begin{tabular}{|l|c|c|c|c|}
\hline & CTL $(\mathrm{n}=7)$ & DNV $(\mathrm{n}=7)$ & ADV $(\mathrm{n}=9)$ & DYS $(\mathrm{n}=11)$ \\
\hline Age (years) & $56.1 \pm 2.98$ & $52.6 \pm 3.13$ & $61.6 \pm 3.56$ & $61.4 \pm 2.84$ \\
\hline Disease duration (years) & NA & $2.30 \pm 0.60^{*}$ & $7.05 \pm 1.55$ & $9.98 \pm 1.73$ \\
\hline H\&Y & NA & $1.14 \pm 0.14^{* *}$ & $2.11 \pm 0.11$ & $2.18 \pm 0.12$ \\
\hline MDS-UPDRS I & NA & $5.00 \pm 2.06$ & $3.25 \pm 0.92$ & $5.00 \pm 1.04$ \\
\hline MDS-UPDRS II & NA & $3.66 \pm 0.80^{\star}$ & $4.75 \pm 1.34$ & $8.36 \pm 1.43$ \\
\hline MDS-UPDRS III & NA & $15.28 \pm 2.17$ & $20.00 \pm 3.33$ & $13.18 \pm 3.34$ \\
\hline MDS-UPDRS IV & NA & NA & $2.00 \pm 1.00$ & $4.36 \pm 0.59$ \\
\hline LEDD (mg) & NA & $57.6 \pm 20.2^{\S \neq}$ & $495.0 \pm 56.4^{\dagger}$ & $846.6 \pm 96.4$ \\
\hline AIMS & NA & NA & NA & $4.36 \pm 0.98$ \\
\hline
\end{tabular}

H\&Y: Hoehn and Yahr staging; MDS-UPDRS: Movement Disorder Society-sponsored revision of the Unified Parkinson Disease Rating Scale; LEDD: Levodopa-equivalent daily dose; CTL: control; DNV: De Novo patients; ADV: Advanced patients; DYS: Dyskinetic patients; NA: not applicable

$* P<0.05$ vs ADV and DYS Mann-Whitney post hoc test; $* * P<0.001$ vs ADV and DYS Mann-Whitney post hoc test; $\downarrow P<0.05$ vs DYS Mann-Whitney post hoc test; $\S P<0.05$ vs ADV Tukey post hoc test; $\ddagger P<0.001$ vs DYS Tukey post hoc test $t_{s} \dagger P<0.05$ vs DYS Tukey post hoc test

Table 2 Subjective measures of depression and sleep quality

\begin{tabular}{l|cccccc}
\hline & CTL $(\mathrm{n}=7)$ & DNV $(\mathrm{n}=7)$ & ADV $(\mathrm{n}=9)$ & DYS $(\mathrm{n}=11)$ & Kruskal-Wallis & P \\
& & & & & $\chi^{2}$ & values \\
\hline mBDI & $5.14 \pm 1.71$ & $8.42 \pm 2.89$ & $5.11 \pm 2.01$ & $9.00 \pm 2.13$ & 3.096 & 0.377 \\
ESS & $5.11 \pm 0.98$ & $7.00 \pm 1.90$ & $7.33 \pm 1.53$ & $8.27 \pm 1.23$ & 2.105 & 0.551
\end{tabular}




\begin{tabular}{l|llllll} 
PSQI & $5.42 \pm 1.19$ & $5.85 \pm 1.47$ & $5.22 \pm 0.87$ & $8.00 \pm 1.03$ & 3.707 & 0.295
\end{tabular}

CTL: control; DNV: De Novo patients; ADV: Advanced patients; DYS: Dyskinetic patients; mBDI: Beck depression scale; ESS: Epworth sleepiness scale;

PSQI: Pittsburgh sleep quality index

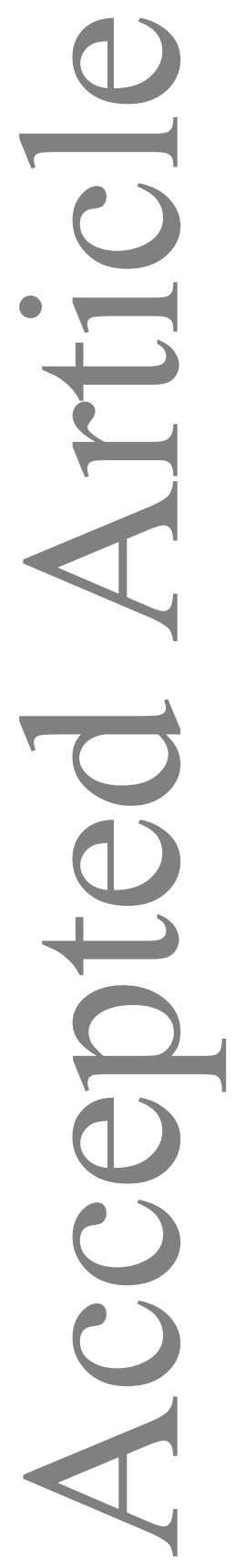


Table 3 Descriptive polysomnographic data

\begin{tabular}{|c|c|c|c|c|c|c|}
\hline & CTL $(\mathrm{n}=7)$ & $\operatorname{DNV}(\mathrm{n}=7)$ & $\operatorname{ADV}(n=9)$ & DYS $(n=11)$ & $\begin{array}{c}\text { Kruskal-Wallis } \\
\chi^{2}\end{array}$ & $\mathrm{P}$ values \\
\hline TST (min) & $297.4 \pm 23.4$ & $351.0 \pm 9.68$ & $309.3 \pm 15.0$ & $271.3 \pm 27.4$ & 5.434 & 0.143 \\
\hline $\mathrm{SL}(\min )$ & $77.88 \pm 28.2$ & $12.32 \pm 2.48$ & $42.28 \pm 18.7$ & $32.45 \pm 13.6$ & & \\
\hline SE & $0.62 \pm 0.05$ & $0.77 \pm 0.29$ & $0.65 \pm 0.45$ & $0.55 \pm 0.05$ & 8.155 & 0.043 \\
\hline WASO (min) & $106.0 \pm 28.9$ & $89.7 \pm 15.5$ & $106.4 \pm 21.7$ & $154.8 \pm 19.9$ & 5.268 & 0.153 \\
\hline Wake $(\%)$ & $25.48 \pm 6.74$ & $19.92 \pm 3.04$ & $24.15 \pm 4.57$ & $36.60 \pm 5.06$ & 4.730 & 0.193 \\
\hline SWS (\%) & $18.52 \pm 1.41$ & $21.84 \pm 4.19$ & $21.87 \pm 5.37$ & $16.95 \pm 2.76$ & 0.648 & 0.885 \\
\hline REM (\%) & $14.04 \pm 3.47$ & $15.37 \pm 2.41$ & $12.82 \pm 2.40$ & $8.40 \pm 2.24$ & 4.648 & 0.198 \\
\hline
\end{tabular}

CTL: control; DNV: De Novo patients; ADV: Advanced patients; DYS: Dyskinetic patients;

TST: total sleep time; SL: sleep latency; SE: sleep efficiency; WASO: wakefulness after sleep onset; SWS: slow wave sleep; REM: rapid eye movement.

Table 4 Descriptive actigraphy data

\begin{tabular}{lcccccc}
\hline & CTL $(\mathrm{n}=7)$ & DNV $(\mathrm{n}=$ & ADV $(\mathrm{n}=$ & $\mathrm{DYS}(\mathrm{n}=$ & Kruskal-Wallis $\chi^{2}$ & P values \\
& & $6)$ & $8)$ & $10)$ & \\
\hline eTST & $348.9 \pm$ & $423.9 \pm 8.6$ & $408.9 \pm$ & $371.0 \pm 14.5$ & 4.823 & 0.183 \\
$(\min )$ & 50.5 & & 24.5 & & & \\
eSL (min) & $21.50 \pm$ & $11.5 \pm 4.29$ & $13.46 \pm$ & $19.06 \pm 4.27$ & 1.953 & 0.582 \\
& 8.85 & & 2.97 & & \\
eSE & $89.78 \pm$ & $88.4 \pm 2.05$ & $83.05 \pm$ & $80.44 \pm 2.67$ & 4.747 & 0.191 \\
& 1.47 & & 3.65 & & & \\
eTB (min) & & & & & 1.282 & 0.733 \\
\hline
\end{tabular}

CTL: control; DNV: De Novo patients; ADV: Advanced patients; DYS: Dyskinetic patients;

eTST: estimated total sleep time; eSL: estimated sleep latency; eSE: estimated sleep efficiency 


\section{Figure Legends}

Fig. 1: Design of the study and segments of nonrapid eye movement (NREM) that were subjected to analysis. A) Schematic flow chart of the study and number of subjects enrolled and tests and/or examinations performed. After the screening visit, participants began the 1-week actigraphy study. Subsequently, the first sleep laboratory habituation night coincided with the last actigraphic day; on the following day, whole-night video polysomnography-high-density EEG (vPSG-hdEEG) recording was performed, and the recordings were assessed and scored. Participants with SRBDs at sleep assessment were excluded from further analysis. Three additional patients were excluded from the SWA analysis for misdiagnosis (suspected Lewy body dementia and multiple system atrophy) or for technical reasons. B) Whole-night NREM sleep was extracted, epochs containing arousals were excluded, and the remainder was subdivided into 10 equal segments. The $2^{\text {nd }}, 3^{\text {rd }}$, and $4^{\text {th }}$ segments and the $7^{\text {th }}, 8^{\text {th }}$, and $9^{\text {th }}$ segments were selected, as early and late sleep, respectively, and were analyzed further.

Fig. 2: Correlation analysis between selected polysomnographic parameters and disease duration. A) Total sleep time (TST) showed a clear negative correlation with disease duration only in the whole pool of patients $\left(\mathrm{n}=27\right.$, Spearman's test, $\left.\mathrm{r}_{\mathrm{s}}=-0.527, P=0.005\right)$, while no correlation was found within individual patient groups. B) Sleep efficiency (SE) was negatively correlated with disease duration in all patients $\left(\mathrm{n}=27\right.$, Spearman's test, $\left.\mathrm{r}_{\mathrm{s}}=-0.659, P<0.001\right)$ and in DYS patients specifically $\left(\mathrm{n}=11\right.$, Spearman's test, $\left.\mathrm{r}_{\mathrm{s}}=-0.664, P=0.026\right)$. C) Regarding the relationship between slow wave sleep (SWS) and disease duration, there was a positive correlation in both DNV ( $\mathrm{n}=7$; Spearman's test, $\left.\mathrm{r}_{\mathrm{s}}=0.786, P=0.036\right)$ and $\mathrm{ADV}(\mathrm{n}=9$; Spearman's test, $\left.\mathrm{r}_{\mathrm{s}}=0.783, P=0.013\right)$ groups, but a negative correlation in DYS patients $(\mathrm{n}=$ 11; Spearman's test, $\left.\mathrm{r}_{\mathrm{s}}=-0.761, P=0.007\right)$. 
Fig. 3: Correlation analysis between selected polysomnographic parameters and levodopaequivalent daily dose (LEDD) in ADV, DYS, and combined ADV and DYS groups. A) Total sleep time (TST) showed a clear negative correlation with LEDD only in ADV+DYS patients (n $=20$; Spearman's test, $\left.\mathrm{r}_{\mathrm{s}}=-0.682, \mathrm{P}=0.021\right)$. B) Sleep efficiency $(\mathrm{SE})$ was negatively correlated with LEDD both in DYS patients $\left(\mathrm{n}=11\right.$, Spearman's test, $\left.\mathrm{r}_{\mathrm{s}}=-0.659, \mathrm{P}<0.001\right)$ and ADV+DYS patients $\left(n=20\right.$, Spearman's test, $\left.r_{s}=-0.664, P=0.026\right)$. C) Slow wave sleep (SWS) was negatively correlated with LEDD only in DYS patients $\left(n=11\right.$, Spearman's test, $r_{s}=-0.682$, $\mathrm{P}=0.021)$.

Fig. 4: Selected actigraphic parameter correlation analysis with the Abnormal Involuntary Movement Scale (AIMS) and Movement Disorder Society-sponsored revision of the Unified Parkinson Disease Rating Scale (MDS-UPDRS) IV in DYS patients. A) The 1-week estimated TST (eTST) was negatively correlated with AIMS and MDS-UPDRS scores (Spearman's test, n $=9 ; \mathrm{r}_{\mathrm{s}}=-0.733, P=0.035 ; \mathrm{r}_{\mathrm{s}}=-0.817, P=0.007$, respectively). B) No correlations of the 1 week estimated SE (eSE) and AIMS or MDS-UPDRS IV scores were found (Spearman's test, $\mathrm{n}$ $=9 ; \mathrm{r}_{\mathrm{s}}=-0.536, P=0.137$ and $\mathrm{r}_{\mathrm{s}}=-0.221, P=0.567$, respectively).

Fig. 5: Slow wave activity during nonrapid eye movement (SWA-NREM) sleep analysis. A) Topography and power maps at 2, 3, and $4 \mathrm{~Hz}$ during early and late sleep in healthy control individuals (CTL) and Parkinson's disease patients at various stages of disease (DNV, ADV, DYS). B) We found a significant difference between early and late sleep in CTL ( $n=7)$, DNV (n $=5)$, and ADV individuals $(\mathrm{n}=9 ; P<0.01)$ but not in DYS patients $(\mathrm{n}=10)$, which remained significant after correction for multiple comparisons. C) Plots of the mean power of high-density (HD, empty dots) or low-density EEG (LD, green dots) in each group of subjects during early 
and late sleep (CTL, DNV, ADV, and DYS). Lines are the mean of all HD (black) or LD (green) channels. All groups, except the DYS group, showed a difference between early and late sleep.

Fig. 6: Changes in slow wave activity (SWA) content in each group of subjects during early and late sleep (CTL, DNV, ADV, and DYS). The SWA content in early (dark-blue line) and late (light-blue line) sleep in each group of subjects by selecting all the high-density EEG (all HD, upper plot) or only the frontal high-density channels (frontal HD, middle plot) for the SWA analysis. In the lower plot, only the frontal low-density EEG channels were analyzed (frontal LD).

$\S P<0.01$ vs CTL; $\# P<0.01$ vs DNV; $\$ P<0.01$ vs ADV; $\uparrow P<0.01$ vs DYS.

Fig. 7: Slow wave activity during nonrapid eye movement (SWA-NREM) sleep source analysis in control individuals (CTL). sLORETA analysis showed a decrease in SWA between early and late sleep, only in the CTL individuals $(\mathrm{n}=7 ; P<0.01$ ), which was localized over the frontocentral regions (Brodmann areas: 4, 6, 13, 24, 31). 
All patients

A

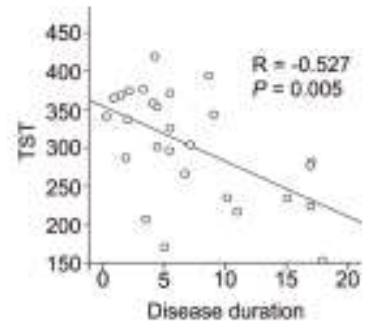

B

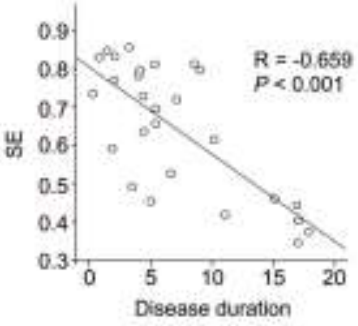

c

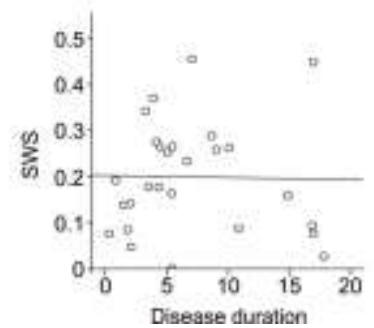

DNV
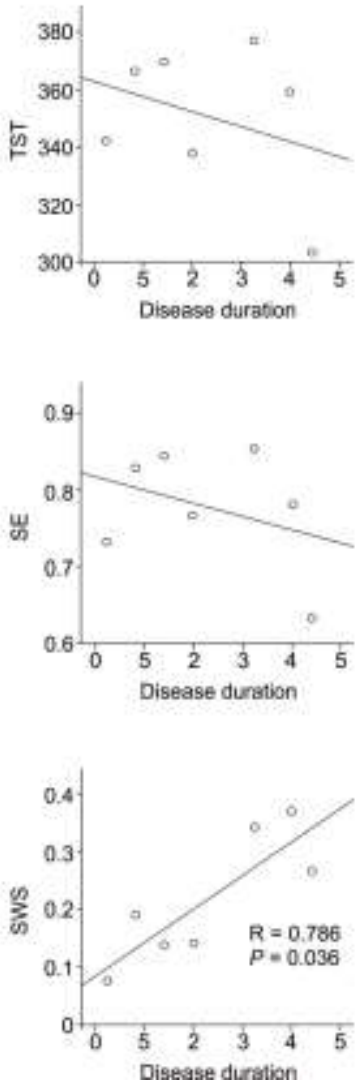

ADV
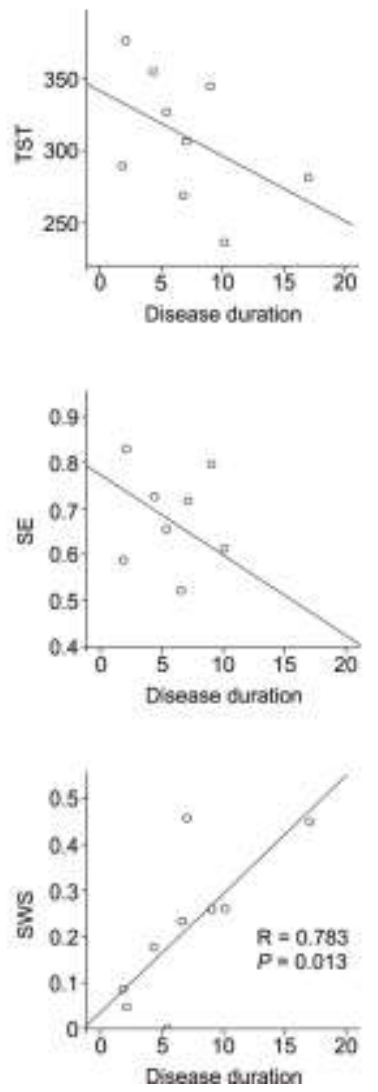

DYS
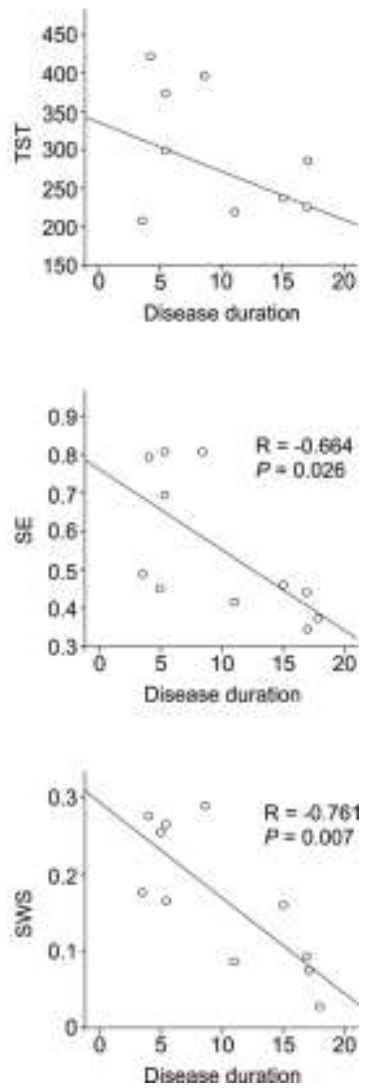

This article is protected by copyright. All rights reserved. 


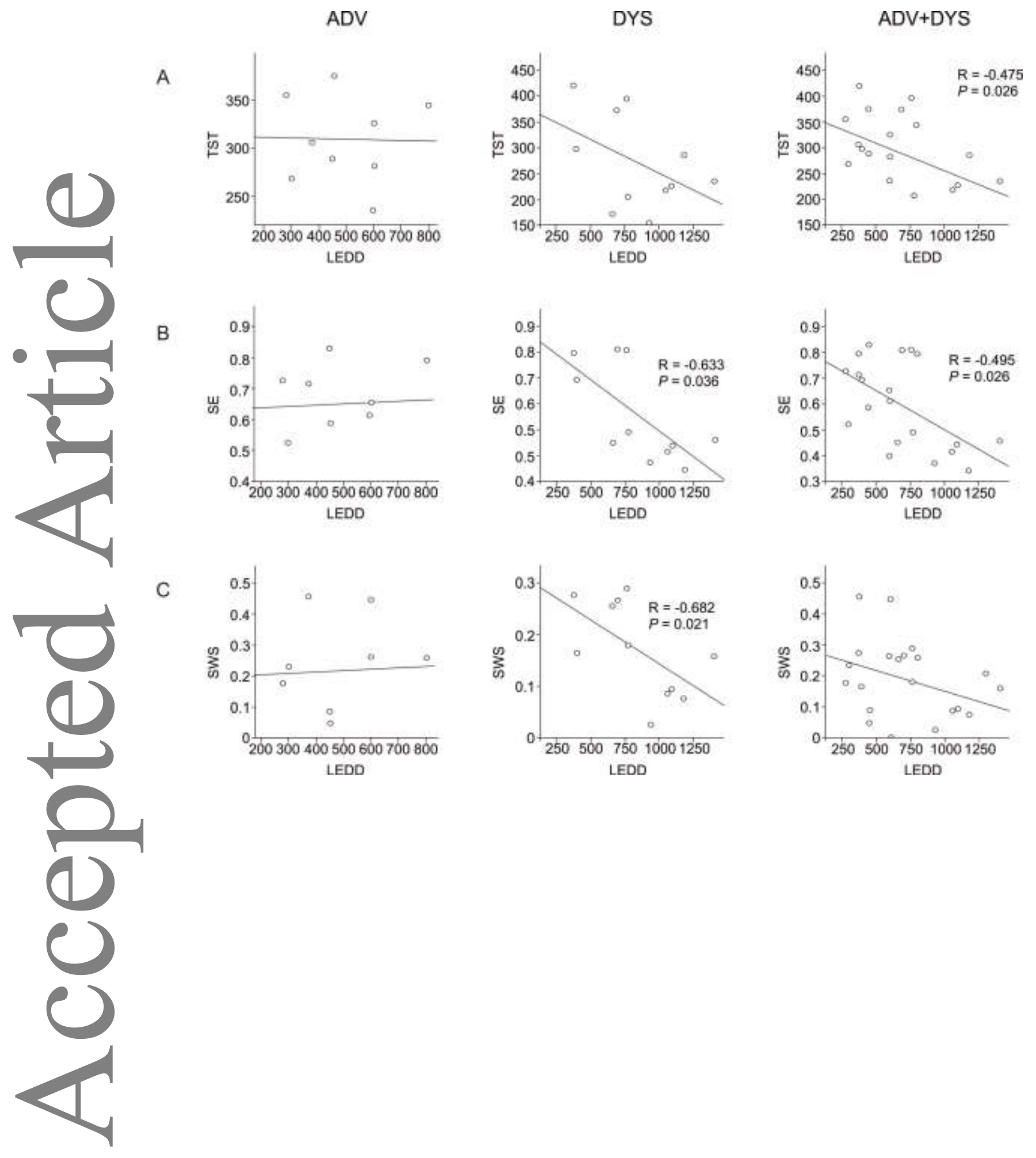

This article is protected by copyright. All rights reserved. 


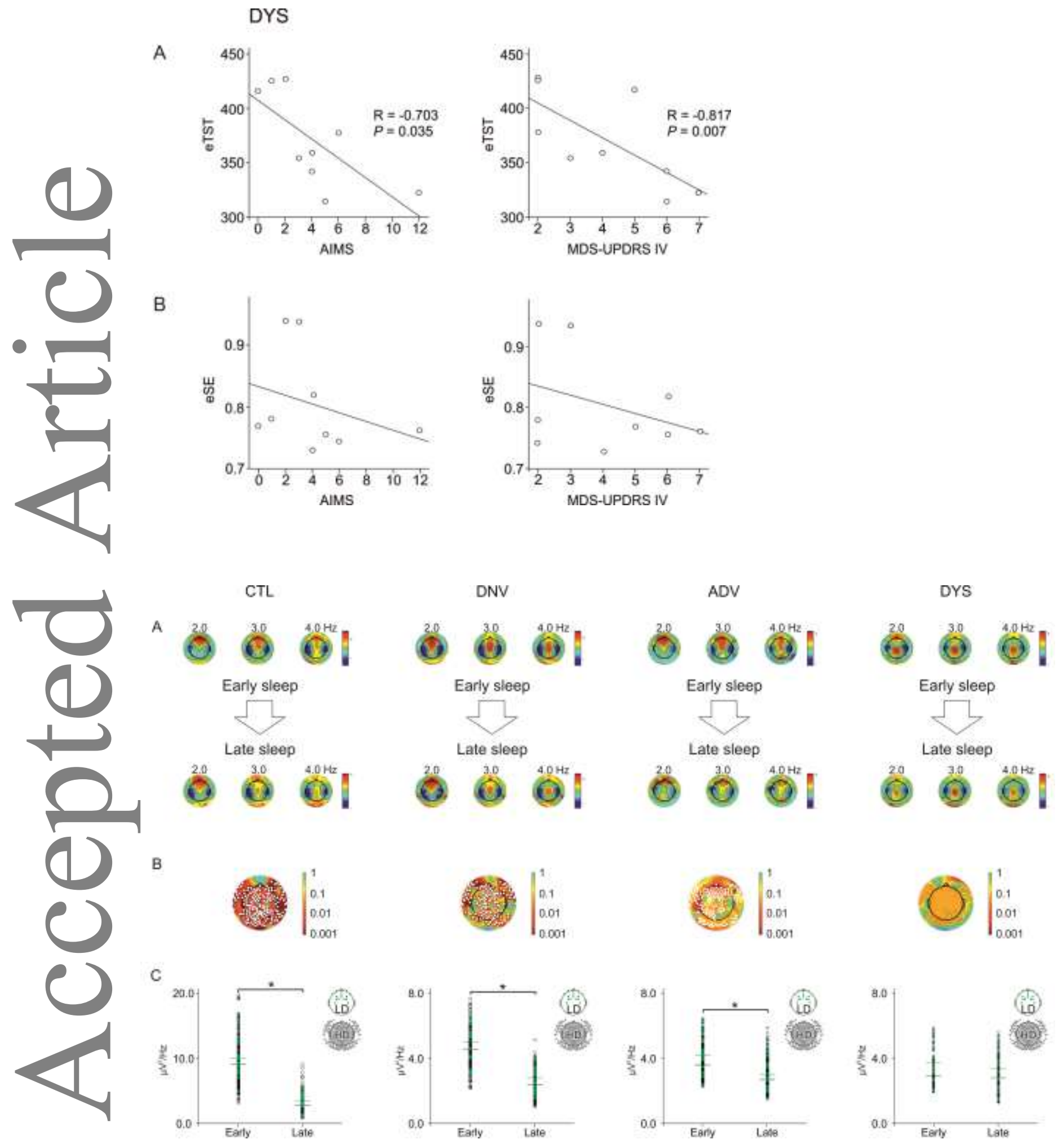




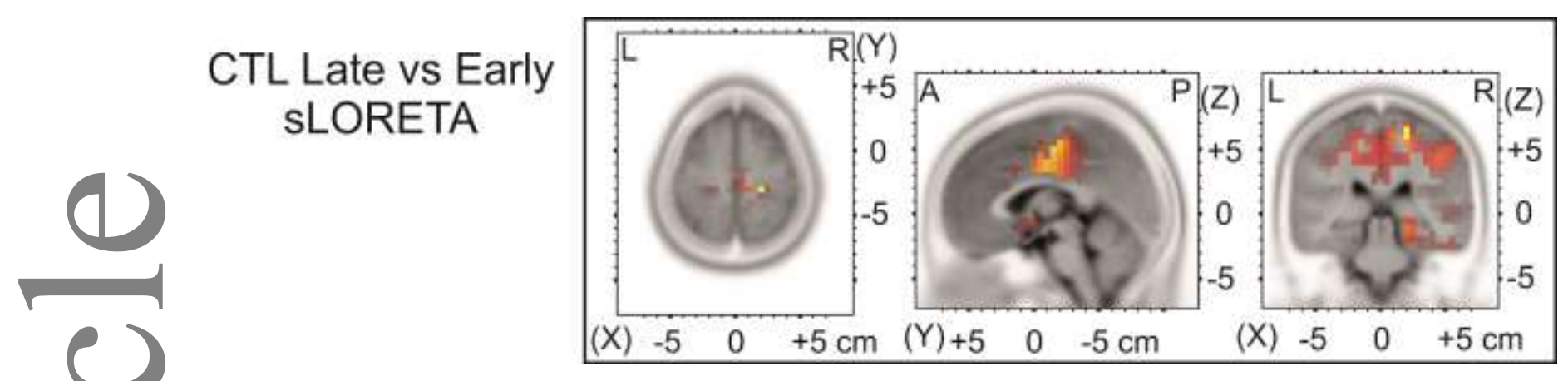

This article is protected by copyright. All rights reserved. 